\title{
IMPORTÂNCIA DA INFLAMAÇÃO NA HIPERTENSÃO ARTERIAL: REVISÃO
}

\author{
GALVÃo, André Luiz Baptista ${ }^{1}$ \\ PALAZZO, Elzylene Léga ${ }^{2}$ \\ PINTO, Mildre Loraine ${ }^{3}$ \\ VIEIRA, Manuela Cristina ${ }^{4}$
}

\begin{abstract}
RESUMO: Componentes do sistema imune inato e adaptativo desempenham uma participação importante na fisiopatologia da hipertensão arterial sistêmica (HAS). A atividade de macrófagos, células dendríticas, linfócitos T e linfócitos B estão associadas a disfunção endotelial e infiltrado inflamatório perivascular nos vasos sanguíneos, nos rins, no encéfalo e coração, alterações estas, intimamente relacionadas com a instalação e desenvolvimento da HAS. A deformação mecânica nas células endoteliais dos vasos sanguíneos, que ocorre durante a elevação da pressão arterial, promove uma cascata de sinalização e eventos que estimulam a liberação e expressão de moléculas de adesão e mediadores inflamatórios que recrutam as células inflamatórias. O óxido nítrico (NO) e as espécies reativas do oxigênio (ERO) são elementos do sistema imune inato, produzidos por macrófagos, que atuam no processo inflamatório desencadeado por alterações mecanoelásticas dos vasos sanguíneos durante a HAS. A resposta imune adaptativa também pode estar presente nestas condições, pois após o aumento da atividade de células da imunidade inata e a presença de ERO, ocorre liberação de mediadores inflamatórios que recrutam as células dendríticas, linfócitos T e linfócitos B. As células inflamatórias e as ERO também sensibilizam o sistema nervoso central (SNC) que resulta em maior atividade do sistema nervoso simpático (SNS) induzindo a vasoconstrição e aumento da volemia. Outros fatores, como a renina, angiotensina II, aldosterona e a liberação de citocinas estão diretamente envolvidas em todos esses processos. Descrever o papel da inflamação na HAS constituiu no objetivo da presente revisão de literatura.
\end{abstract}

Palavras-chaves: Coração. Linfócitos. Macrófagos. Rins

\section{IMPORTANCE OF INFLAMMATION IN HYPERTENSION - REVIEW}

\begin{abstract}
SUMMARY: Components of the innate and adaptive immune system play an important role in the pathophysiology of hypertension (HBP). The activity of macrophages, dendritic cells and lymphocytes are associated with endothelial dysfunction and perivascular inflammatory infiltrate in the blood vessels, kidneys, brain and heart, these changes, closely related to the installation and development of hypertension. The mechanical deformation in endothelial cells of blood vessels that occurs during the elevation of blood pressure, causes a cascade of signaling events and stimulating the expression and release of adhesion molecules and inflammatory mediators that recruit inflammatory cells. Nitric oxide (NO) and reactive oxygen species (ROS) are elements of the innate immune system, produced by macrophages, which act in the inflammatory process triggered by changes deformation in endothelial cells of blood vessels during hypertension. The adaptive immune response may also be present in these conditions, because after the increased activity of cells of innate immunity and the presence of ROS, occurs release of inflammatory mediators that recruit dendritic cells and lymphocytes. Inflammatory cells and ROS also sensitize the central nervous system (CNS) that results in increased activity of the sympathetic nervous system (SNS) inducing vasoconstriction and increased blood volume. Other factors, such as renin, angiotensin II, aldosterone and the release of cytokines are directly involved in all these processes. Describe the role of inflammation in hypertension the purpose of this literature review.
\end{abstract}

Keywords: Heart. Lymphocytes. Macrophages. Kidney

\footnotetext{
${ }^{1}$ Doutorando em Medicina Veterinária da Faculdade de Ciências Agrárias e Veterinárias Universidade Estadual Paulista campus Jaboticabal (SP).Email: andrelgalvao@ hotmail.com.

${ }^{2}$ Docente da Faculdade Dr. Francisco Maeda - Fundação Educacional de Ituverava (SP).

${ }^{3}$ Docente do Centro Universitário Moura Lacerda; Centro Universitário Barão de Mauá, Ribeirão Preto (SP).

${ }^{4}$ Pós-doutoranda em Medicina Veterinária da Faculdade de Ciências Agrárias e Veterinárias Universidade Estadual Paulista campus Jaboticabal (SP).
} 


\section{INTRODUÇÃO}

O endotélio vascular é uma monocamada de epitélio pavimentoso que reveste internamente os vasos sanguíneos e faz interface entre o sangue e músculo liso vascular, sendo responsável pelo tônus vascular, controlando as respostas vasodilatadoras ou vasoconstritoras locais, em resposta a alterações do fluxo sanguíneo e aos agentes vasoativos (CERAVOLO et al., 2012).

Entende-se por disfunção endotelial por um desequilíbrio na produção endotelial de mediadores que regulam o tônus vascular, agregação plaquetária, coagulação e fibrinólise, sendo o tônus vascular o aspecto mais estudado (CERAVOLO et al., 2012).

Alterações nos vasos sanguíneos de seres humanos e animais com hipertensão arterial sistêmica (HAS), estão associadas a múltiplos fatores, dentre eles a inflamação é evidenciada. Os componentes do sistema renina angiotensina aldosterona (SRAA) funcionam como agentes próinflamatórios que participam da inflamação vascular (SAVOIA; SCHIFFRIN, 2006; HARRISON, 2013; SANTISTEBAN et al., 2013).

O coração, os rins, e o encéfalo constituem em órgãos alvos da HAS, as células inflamatórias, como macrófagos, monócitos, células dendríticas se acumulam nos vasos sanguíneos, mais precisamente na camada adventícia e no tecido adiposo perivascular durante a instalação e progressão da HAS (HARRISON et al., 2012; SCHIFFRIN, 2013).

A pressão necessária para mover o sangue através do sistema circulatório é provida pela ação de bomba do coração (débito cardíaco) e pela tensão (tônus) vascular, especialmente das arteríolas (CONSOLIM-COLOMBO et al., 2012). Pequenas reduções do lúmen das pequenas artérias aumentam significativamente a resistência vascular periférica durante a HAS, estando à inflamação envolvida neste processo remodelação vascular que compromete o sistema cardiovascular (SAVOIA; SCHIFFRIN, 2006).

No exame histopatológico renal de pacientes com hipertensão maligna, notadamente observa-se o infiltrado de células inflamatórias (HARRISON et al., 2011). O infiltrado de macrófagos e células T nos glomérulos de animais e humanos promove danos aos rins, com lesão no endotélio vascular e nos podócitos (HARRISON et al., 2012). Os macrófagos possuem uma participação importante na inflamação do sistema cardiovascular, não somente nos rins, pois está envolvido na fibrose e remodelamento das células cárdicas (BROWN, 2013). O encéfalo também é um sítio de inflamação no desenvolvimento da HAS, as células da microglia, que são as células do sistema imune do sistema nervoso central (SNC) podem desenvolver propriedades similares de macrófagos ativados, estas células participam do controle da pressão arterial (PA), devido a 
ação da angiotensina II estas funções podem ser comprometidas, podendo promover o aumento da atividade do sistema nervoso simpático (SNS) e aumento da PA (HARRISON et al., 2012).

Espécies reativas do oxigênio (ERO) e óxido nítrico (NO) são componentes fundamentais da resposta imune inata, entretanto na ocorrência de lipoperoxidação de proteínas, podem formar neoantígenos que ativam a resposta imune adaptativa, provocando uma variedade de respostas inflamatórias que desempenham um papel importante no sistema cardiovascular (HARRISON et al., 2011). Descrever a participação do processo inflamatório na HAS constituiu no objetivo da presente revisão de literatura.

\section{COMPONENTES DO SISTEMA IMUNOLÓGICO}

A primeira forma de defesa contra patógenos é a resposta imune inata, os seus componentes incluem células epiteliais, macrófagos, neutrófilos e sistema complemento. O NO e as ERO são componentes da resposta imune inata, e são produzidos por macrófagos modulando a atividade, função e sobrevivência de linfócitos T, linfócitos B e células dendríticas. (HARRISON et al., 2011; SCHIFFRIN, 2013; SANTISTEBAN et al., 2013).

As células endoteliais, assim como os demais tipos celulares da parede vascular, produzem ERO, como o ânion superóxido e o peróxido de hidrogênio, elas também sintetizam o NO e peroxinitrito, estes radicais livres estão envolvidos em diversos mecanismos benéficos e/ou prejudiciais no organismo (CERAVALO et al., 2012).

Quando ativadas as células endoteliais induzem ao aumento de citocinas inflamatórias (interleucina -8, dentre outras) e moléculas de adesão ( $\mathrm{P}$ e E-selectina, molécula de adesão intercelular - ICAM - e molécula de adesão vascular - VCAM) que são o gatilho para adesão e migração de leucócitos para o espaço subendotelial, processo fundamental para o início e progressão do processo inflamatório vascular (CHAVES-JUNIOR, 2012).

Os macrófagos estão sujeitos a responderem a qualquer estímulo, tais como em condições de dano tecidual induzido pela angiotensina II. Estas células são uma fonte potencial de ERO, estando presentes em todos os fenômenos inflamatórios durante a HAS, os produtos produzidos pela inflamação como citocinas podem difundir para células adjacentes do endotélio e da musculatura lisa, promovendo hiperplasia e hipertrofia vascular. Os macrófagos também participam da apresentação de antígenos aos linfócitos T, sendo esta célula também importante para a instalação do processo inflamatório durante a HAS (HARRISON et al., 2012; SCHIFFRIN, 2013).

O sistema imune adaptativo também participa da gênese da HAS, pois em decorrência de 
diversos estímulos ocasionados pela hipertensão, proteínas endógenas são modificadas e podem se transformar em neoantígenos. Este argumento é justificado pelo conhecido papel da lesão oxidativa, que provavelmente modifica as proteínas endógenas e as transforma em neoantígenos (HARRISON et al., 2011; SCHIFFRIN, 2013). Neste sentido, as células apresentadores de antígenos como as células dendríticas, também podem participar do processo inflamatório durante a HAS, entretanto a participação deste grupo de celular não está bem elucidado ainda, mas acredita-se que elas estejam envolvidas na captação de neoantígenos e os apresentem aos linfócitos T durante a HAS (HARRISON et al., 2012; SCHIFFRIN, 2013). De acordo com Brown (2013) os receptores mineralocorticóides e a aldosterona modulam a interação entre as células apresentadoras de antígeno e linfócitos T, por meio da liberação da citocinas como a IL-6 e fator de crescimento tumoral - beta 1 (TGF- $\beta 1)$.

Os linfócitos $\mathrm{T}$ se tornam mais ativos e presentes na circulação sanguínea de pacientes hipertensos, estas células se acumulam na camada adventícia dos vasos sanguíneos, bem como, no tecido adiposo perivascular, principalmente dos rins (HARRISON et al., 2011; HARRISON et al., 2012; SCHIFFRIN, 2013). Uma das citocinas mais importantes liberadas pelos linfócitos T durante a HAS trata-se da IL-17, pois este componente inflamatório estimula a quimiotaxia de outras células inflamatórias, nos vasos sanguíneos durante a HAS (HARRISON et al., 2011; HARRISON et al., 2012; SCHIFFRIN, 2013). Outro fator importante para o aumento da expressão da IL-17 é a IL-6 que é sintetizada pelos linfócitos T, linfócitos B, fibroblastos e células endoteliais. A IL-6 estimula o fígado na produção de proteínas de fase aguda, incluindo a proteína amilóide sérica A, proteína $\mathrm{C}$ reativa e a redução da albumina (HARRISON et al., 2012). A IL-6 também participa da gênese da HAS, conforme demonstrado que alguns feocromocitomas que causam HAS grave produzem a IL-6 (HARRISON et al., 2012). Zhang et al. (2012) demonstraram que o acúmulo de IL-6 em pacientes com doença renal crônica (DRC) hipertensos foi significativamente maior quando comparados aos pacientes com DRC não hipertensos. A angiotensina II em interação com o receptor AT1 estimula a produção de IL-6 pelas células da musculatura lisa do tecido vascular (HARRISON et al., 2012).

As células endoteliais também podem atuar no inicio do processo inflamatório nos vasos sanguíneos durante a HAS, pois promovem adesão e permite infiltração de macrófagos por um mecanismo dependente dos receptores mineralocorticóides, bem como a aldosterona que estimula a expressão de ICAM-1 que promove a adesão de linfócitos no endotélio vascular (BROWN, 2013). 


\section{INFLAMAÇÃO E SISTEMA RENINA ANGIOTENSINA ALDOSTERONA}

A ativação do SRAA desempenha um papel importante no desenvolvimento e na fisiopatologia de doenças do sistema cardiovascular, em especial na HAS (SAVOIA; SCHIFFRIN, 2006). A angiotensina II atua como potente agente pró-inflamatório, modulando repostas com quimiotaxia, proliferação e diferenciação de monócitos em macrófagos, através do aumento da expressão de moléculas de adesão e citocinas, induzindo a adesão de células inflamatórias às células endoteliais. Provavelmente estes efeitos inflamatórios são mediados pela ativação do fator nuclear kappa $\beta$ (NF-K $\beta$ ), o qual induz a expressão de genes pró-inflamatórios, como IL-6, VCAM-1 e proteína de atração de monócito (MCP-1) (RIGATTO et al., 2004; CAMPBELL, 2013).

Nos vasos sanguíneos, a ativação dos receptores AT1 pela angiotensina II, promove aterogênese e induz o estresse oxidativo, provocando a formação de ERO como superóxido e peróxido de hidrogênio pela ativação da NADH/NADPH oxidases. Resultando em hiperplasia e hipertrofia das células da musculatura lisa dos vasos. Neste contexto, o SRAA possui o envolvimento na modulação e biodisponibilidade do NO por meio da estimulação a produção de ânion superóxido. A diminuição do NO é descrita em várias formas de lesão vascular e disfunção endotelial, devido à hipertensão (RIGATTO et al., 2004; CERAVOLO et al., 2012; CAMPBELL, 2013).

As células endoteliais estão dispostas de modo estratégico na parede do vaso, recebendo sinais hemodinâmicos e humorais, bem como desempenham respostas efetoras adaptativas locais. Em situação de estresse oxidativo, principalmente com a participação do ânion superóxido ocorre o processo de disfunção endotelial, que reduz a disponibilidade de $\mathrm{NO}$, deste modo o NO é retirado pela reação com o ânion superóxido produzindo a molécula peroxinitrico, que é altamente deletéria e capaz de reduzir os efeitos vasodilatadores, antiproliferativos, antiinflamatório e antiaterogênico do NO (RIGATTO et al., 2004; SCHIFFRIN, 2013).

A interação da angiotensina II e seu receptor AT1 modulam a resposta inflamatória envolvida com estresse oxidativo, podendo promover efeitos cronotrópicos e inotrópicos positivos no coração, induzir hipertrofia e apoptose das células cardíacas (DORN, 2009). A administração da angiotensina II por via subcutânea em camundongos demonstrou hipertrofia da camada média da artéria aorta e infiltração perivascular por linfócitos $\mathrm{T}$ e macrófagos, com conseqüente espessamento da parede vascular, bem como lesão aos miócitos e fibrose intersticial em células cardíacas podem estar presentes. Entretanto, quando administrada pela via intravenosa a angiotensina II pode estar relacionada com a HAS, lesão glomerular, proteinúria e fibrose intersticial no tecido renal (CAMPBELL, 2013). 
Ganta et al. (2005) e Santisteban et al. (2013) reportaram que a administração de angiotensina II, aumenta a atividade do SNS do baço, gânglios linfáticos e da medula óssea, que por sua vez aumenta a atividade e liberação de linfócitos T circulantes e mediadores inflamatórios como IL-1, IL-2, IL-6 e IL-16 na circulação, resultando em disfunção endotelial e inflamação vascular.

Não somente a angiotensina II está relacionada com instalação do processo inflamatório e estresse oxidativo na HAS, estudos demonstraram que a aldosterona pode promover a liberação de mediadores inflamatórios, como a ciclo-oxigenase-2, proteína quimiotática de monócitos-1 (MPC-1) e ICAM-1, recrutando monócitos e macrófagos nos vasos sanguíneos do coração e dos rins, com conseqüente fibrose tecidual (BROWN, 2013; SCHIFFRIN, 2013).

A aldosterona contribui para a instalação de estresse oxidativo através do aumento da atividade de macrófagos nas células mesangiais, podócitos, células endoteliais do coração e da artéria aorta, bem como pelo aumento da atividade enzima NADPH, que sua vez, estimula a ativação da angiotensina II, condições estas descritas que predispõe a disfunção endotelial, embora, a aldosterona também esteja relacionada com um maior comprometimento das células endoteliais através do aumento e a rigidez destas células (BROWN, 2013).

O estimulo da liberação e/ou expressão da endotelina-1 pode ser induzido pela angiotensia II e pela aldosterona, e contribui para a fibrose do tecido cardíaco, devido ao aumento da síntese de colágeno pelos fibroblastos no coração (BROWN, 2013). A endotelina-1 por si só, trata-se de um importante mediador do processo inflamatório no sistema cardiovascular, pois está envolvida com a liberação de IL-6 e ICAM-1 (SAVOIA; SCHIFFRIN, 2006).

\section{INFLAMAÇÃO E ESTRESSE OXIDATIVO}

Muitos fatores comuns aos indivíduos hipertensos, como a angiotensina II, aldosterona, citocinas, alterações mecanoelásticas do vaso sanguíneo, síntese de ERO e NO se relacionam e contribuem para a HAS. A produção de ERO pode atuar no SNC que induz o aumento da atividade do SNS, as ERO também provocam alterações nos vasos sanguíneos, e ambos os estímulos promovem a vasoconstrição. Enquanto que nos rins as ERO causam a retenção de sódio e água, com aumento da volemia. Estando o processo inflamatório envolvido em todos estes eventos (HARRISON et al., 2011). As ERO participam da disfunção endotelial, vasoconstrição, hiperplasia e hipertrofia da musculatura lisa dos vasos sanguíneos e apoptose celular (HARRISON, 2013).

Griendling et al.(1994), Savoia e Schiffrin (2006) e Harrison (2013) descreveram que atividade da enzima NADPH oxidase nas células da musculatura lisa e nas células endoteliais, 
são sítios potenciais na produção de ERO e que a angiotensina II, aldosterona, catecolaminas e a endotelina-1 podem ativar a atividade desta enzima, participando da hiperplasia e hipertrofia do músculo liso dos vasos sanguíneos e elevação da PA.

Eventos de oxidação resultam em liberação de mediadores inflamatórios e citocinas pelas células endoteliais promovendo a quimiotaxia e a infiltração de células inflamatórias, uma vez que as células inflamatórias adentram o tecido, o local se torna uma fonte na produção de ERO, levando a uma amplificação do estresse oxidativo nos tecidos adjacentes (HARRISON, 2013).

\section{INFLAMAÇÃO E LESÃO EM ÓRGÃOS ALVO}

No coração o processo inflamatório e os fatores tróficos mediado pela angiotensina II, aldosterona e noradrenalina, agem promovendo a proliferação de fibroblastos e miofibroblastos, seguida da formação de colágeno e fibrose intersticial, ocasionando hipertrofia do miócito e da matriz extracelular. $\mathrm{O}$ aumento do tecido fibroso determina da redução da complacência ventricular, aumenta a distância capilar-miócito e, portanto representa mais um elemento responsável pela hipóxia, dificultando também a transmissão do impulso elétrico nas células cardíacas (CAMPANA et al., 2012; BROWN, 2013; SCHIFFRIN, 2013).

A produção de angiotensina II intrarrenal foi demonstrada, nas células epiteliais tubulares, macrófagos e fibroblastos, sendo também expressa pelas células justaglomerulares e células intersticiais, estas células desempenham um papel importante no processo inflamatório e lesão renal progressiva (NORONHA et al., 2002). A liberação de mediadores inflamatórios pela angiotensina II, aldosterona e endotelina-1 promove disfunção mitocondrial nas células do túbulo contorcido proximal, resultando em estímulo da produção de colágeno que excede a degradação pelas metaloproteinases resultando em fibrose tecidual, também estão associadas a lesão glomerular caracterizada pelo enrugamento dos capilares glomerulares e acúmulo de matriz mesangial, que evoluem para colapso de áreas do glomérulo e fibrose progressiva. As alterações tubulointersticiais são caracterizadas por atrofia tubular, fibrose intersticial e processo inflamatório crônico (ALMEIDA et al., 2012; BROWN, 2013).

Na presença da lesão renal, o exame de fundo de olho deverá mostrar esclerose arteriolar (tortuosidade das arteríolas), bem como pode estar presente lesões na retina (exsudatos algodonosos e hemorragias), lesões estas decorrentes da HAS (ALMEIDA et al., 2012).

A HAS faz aumentar a tensão mecânica nas paredes das arteríolas, provocando estiramento de suas estruturas, principalmente no endotélio, no SNC favorece a ocorrência de acidentes vasculares cerebrais (AVC) (ZATZ et al., 2012). 
A inflamação no SNC foi demonstrada durante a hipertensão em ratos naturalmente hipertensos, com a expressão da molécula de adesão juncional -1 (JAM-1) expressa nas células endoteliais no NTS, com o recrutamento de leucócitos (WAKI et al., 2007).

Em humanos hipertensos, foi observado o aumento do espaço de Virchow-Robin, local este pré-linfático com riqueza em células do sistema imune que permite a comunicação entre do SNC e o SNP (MARIN-PADILLA; KNOPMAN, 2011).

\section{MARCADORES DE INFLAMAÇÃO}

A proteína $\mathrm{C}$ reativa trata-se de um biomarcador de inflamação mais disponível em bem caracterizado, sendo utilizado como um importante marcador de doenças cardiovasculares, especialmente para HAS. A proteína C reativa é sintetizada pelo fígado, na fase aguda do processo inflamatório, mas também é produzida pelas células da musculatura lisa de vasos (SAVOIA; SCHIFFRIN, 2006; HARRISON et al., 2011; SANJULIANI; TORRES, 2012).

A importância fisiopatológica da proteína C reativa, não está totalmente compreendida, entretanto, é sabida que esta proteína é um fator de risco ou mediador de doenças cardiovasculares, sendo mais que um marcador inflamatório, pois está envolvida no processo inflamatório vascular atuando no recrutamento de células inflamatórias (monócitos) e interagindo com angiotensina II, endotelina-1 e produção de ERO, bem como, a proteína $\mathrm{C}$ reativa pode atuar como modulador do processo inflamatório, com ação antiinflamatória (SAVOIA; SCHIFFRIN, 2006). A IL-6 estimula o fígado produzir proteínas de fase aguda, incluindo a elevação da proteína C reativa e amilóide sérica A, e redução da albumina (NISHIMOTO, 2010).

A proteína $\mathrm{C}$ reativa pode ser quantificada por vários métodos já padronizados e validados, com baixo custo (SANJULIANI; TORRES, 2012).

Martínez-Subiela et al., (2013) estudaram o comportamento da proteína C-reativa na urina de cães com leishmaniose com diferentes graus de comprometimento de função renal, e observaram que na presença de proteinúria e com sua maior intensidade ocorre o aumento proporcional da proteína C-reativa urinária, considerando esta proteína como um possível marcador para avaliar danos renais associados a leishmaniose.

Hrovat et al., (2013) estudaram o comportamento da proteína C-reativa na urina de cães envenenados por veneno de serpentes, e reportaram o aumento da proteína C-reativa vinte e quatro horas após ao envenenamento.

\section{INIBIDORES DA ENZIMA CONVERSORA DE ANGIOTENSINA}

O bloqueio do SRAA em pacientes hipertensos resulta em diminuição da disfunção 
endotelial e de marcadores do processo inflamatório, bem como promovem a redução dos marcadores do estresse oxidativo (SAVOIA; SCHIFFRIN, 2006).

Campbell (2013) descreveu o efeito antiinflamatório dos inibidores da ECA justamente por inibir a ação da angiotensina II, consequentemente ocorre a inibição da atividade próinflamatória deste hormônio. Imakiire et al. (2007) demonstraram em pacientes humanos que os inibidores da ECA reduzem o infiltrado de macrófagos nos glomérulos.

\section{SISTEMA NERVOSO PARASSIMPÁTICO}

Estímulos no nervo vago são efetivos no controle do processo inflamatório, pois reduzem a liberação nos tecidos periféricos de citocinas (TNF- $\alpha$, IL-6 e IL-1), bem como as fibras aferentes do nervo vago são capazes de detectar a presença do processo inflamatório nos tecidos. A inervação parassimpática no baço, gânglios linfáticos e da medula óssea reduzem a liberação de células inflamatórias na circulação. Desse modo, o efeito antiinflamatório parassimpático pode ser considerado como um potencial alvo terapêutico em indivíduos hipertensos (SANTISTEBAN et al. 2013).

\section{CONSIDERAÇÕES FINAIS}

Uma deformação mecânica nas células endoteliais dos vasos sanguíneos que ocorre durante a elevação da PA promove uma cascata de sinalização e eventos que estimulam a liberação e expressão de moléculas de adesão e mediadores inflamatórios que recrutam as células inflamatórias. Além do papel muito bem conhecido do SRAA da fisiopatologia da HAS, este sistema possui uma participação fundamental na sinalização e na cascata de eventos do processo inflamatório, estando associado a ação da angiotensina II e aldosterona e seus respectivos receptores. Neste sentido, o SRAA está diretamente relacionado com a disfunção endotelial, hiperplasia e hipertrofia celular nos vasos sanguíneos. Estas condições proporcionam a instalação e progressão de doenças cardiovasculares, em especial a HAS. O conhecimento da inflamação, além de permitir um melhor entendimento da fisiopatologia da HAS, permite o avanço nos estudos voltados para novos métodos precoces em diagnóstico como a utilização de marcadores de inflamação, bem como, no desenvolvimento de alternativas terapêuticas para o controle HAS. 


\section{REFERÊNCIAS}

ALMEIDA, F. A.et al. Acometimento renal na hipertensão arterial. In: BRANDÃO, A. A.; AMODEO, C.; NOBRE, F. Hipertensão. 2. ed. Rio de Janeiro: Elsevier, 2012, cap, 25, p. 181$187,511 \mathrm{p}$.

BROWN, N. J. Contribution of aldosterone to cardiovascular and renal inflammation and fibrosis. Nature reviews practice - Nephrology, London, v. 9, n. 8, p. 459-468, 2013.

CAMPANA, E. M. G.et al. Acometimento cardíaco na hipertensão arterial. In: BRANDÃO, A. A.; AMODEO, C.; NOBRE, F. Hipertensão. 2. ed. Rio de Janeiro: Elsevier, 2012, cap, 24, p. 173-181, 511p.

CAMPBELL, D. J. Evolving concepts of the renin - angiotensina system: Do intravenous and subcutaneous angiotensina II increased blood pressure by different mechanisms? Clinical and experimental pharmacology and physiology, Oxford, v. 40, n. 8, p. 560-570, 2013.

CERAVOLO, G. S.et al. Papel dos principais componentes na gênese da hipertensão arterial: endotélio vascular. In: BRANDÃO, A. A.; AMODEO, C.; NOBRE, F. Hipertensão. 2. ed. Rio de Janeiro: Elsevier, 2012, cap, 10, p. 79-89, 511p.

CHAVES-JUNIOR, H. C. Avaliação clínica e complementar básica. In: BRANDÃO, A. A.; AMODEO, C.; NOBRE, F. Hipertensão. 2. ed. Rio de Janeiro: Elsevier, 2012, cap, 22, p. 159$164,511 \mathrm{p}$.

CONSOLIM-COLOMBO, F. M.; IRIGOYEN, M. C.; KRIEGER, E. M. Papel dos principais componentes na gênese da hipertensão arterial: Sistema Nervoso Simpático. In: BRANDÃO, A. A.; AMODEO, C.; NOBRE, F. Hipertensão. 2. ed. Rio de Janeiro: Elsevier, 2012, cap, 7, p. 53$62,511 \mathrm{p}$.

DORN, G. W. Novel pharmacotherapy's to abrogate posinfartion ventricular remodeling. Nature Reviews Cardiology, London, v. 6, n. 4, p. 283-291, 2009.

GANTA, C. K.et al. Central angiotensina II-enhanced splenic cytokine gene expression in mediated by the sympathetic nervous system. American Journal Physiology. Heart and Circulatory Physiology, Bethesda, v. 58, n. 4, p. H1683-H1691, 2005.

GRIENDLING, K. K.et al. Angiotensin II stimulates NADH and NADPH oxidase activity in cultured vascular smooth muscle cells. Circulation Research, Baltimore, v.74 n. 6, 1141-1148, 1994.

HARRISON, D. G.et al. Inflammation, Immunity, and Hypertension. Hypertension, Dallas, v. 57, p. 132-140, 2011.

HARRISON, D. G.; MARVAR, P. J.; TITZE, J. M. Vascular inflammatory cells in hypertension. Frontiers Research Foundation, Switzerland, v. 3, n. 7, p. 1-11, 2012.

HARRISON, D. G. The mosaic theory revisited: common molecular mechanisms coordinating diverse organ and cellular events in hypertension. Journal of the American Society of Hypertension, New York, v. 7, n. 1, p. 68-74, 2013. 
HROVAT, A.et al. Evaluation of snake envenomation-induced renal dysfunction in dogs using early urinary biomarkers of nephrotoxicity. The Veterinary Journal, New York, v. 6, m. 30, p. $1-6,2013$.

IMAKIIRE, T.et al. Effects of renin-angiotensin system blockade on macrophage infiltration in patients with hypertnesive nephrosclerosis. Hypertension, Dallas, v. 30, p. 635-642, 2007.

MARIN-PADILLA, M.; KNOPMAN, D. Developmental aspects of the intracerebral microvasculature and perivascular spaces insights into brain response to late-life disease. Journal of neuropathology and experimental neurology, New York, v. 70, n. 12, p. 1060-1069, 2011.

MARTÍNEZ-SUBIELA, S.et al.. Urinary C reactive levels in dogs with leishmaniasis at different stages of renal damage. Research in Veterinary Science, London, v. 7, n. 2, p. 1-6, 2013.

NISHIMOTO, N. Interleucin-6 as a therapeutics target in candidate. Clinical Pharmacology \& Therapeutics, Bethesda, v. 87, p. 483-487, 2010.

NORONHA, I. L.; FUJIKARA, C. K.; ZATZ, R. The inflammatory component in progressive renal disease - are interventions possible? Nephrology Dialysis Transplantation, Oxford, v. 17, p. 363-638, 2002.

RIGATTO, K, V.; BÖHLKE, M. IRIGOYEN, M. C. Sistema Renina Angiotensina: da Fisiologia ao Tratamento. Revista da Sociedade Brasileira de Cardiologia do Rio Grande do Sul, Porto Alegre, Ano XIII, n 3, 2004.

SANJULIANI, A. F.; TORRES, M. R. S. Avaliação clínica e complementar diferenciada. In: BRANDÃO, A. A.; AMODEO, C.; NOBRE, F. Hipertensão. 2. ed. Rio de Janeiro: Elsevier, 2012, cap, 23, p. 165-172, 511p.

SANTISTEBAN, M. M.et al. Dysfunction brain-bone marrow communication: a paradigm shift in the pathophysiology of Hypertension, Current Opinion in Nephrology and Hypertension, London, v. 15, p. 377-389, 2013.

SAVOIA, C.; SCHIFFRIN, E. Inflammation in hypertension, Current Opinion in Nephrology and Hypertension, London, v. 15, p. 152-158, 2006.

SCHIFFRIN, E.L.; LIPMAN, M.L.; MANN, J.F.E. Chronic Kidney Disease. Circulation Research, Dallas, v. 116, p. 85-97, 2007.

WAKI, H.et al. Junctional adhesion molecule-1 is up regulated in spontaneously hypertensive rats: evidence for a prohypertensive role within te brain Stem, Hypertension, Dallas, v. 49, n. p. 1321-1327, 2007.

ZATZ, R.; SEGURO, A. C.; MALNIC, G. Bases Fisiológicas da Nefrologia. São Paulo: Atheneu, 2012. 394p.

ZHANG, W.et al. Interleukin-6 underlies angiotensina II- induced hypertension and chronic renal damage. Hypertension, Dallas, v. 59, p. 136-144, 2012. 
